\title{
Simple action planning can affect attentional allocation in subsequent visual search
}

\author{
Seohee Han ${ }^{1}$ - Eunhee $\mathrm{Ji}^{1}$ • Eunhye Choe ${ }^{1}$ - Dogyun Kim ${ }^{1}$ • Min-Shik Kim ${ }^{1}$
}

Published online: 17 June 2020

(C) The Psychonomic Society, Inc. 2020

\begin{abstract}
Previous research has shown that mental representations of actions can influence performance on relevant tasks or dimensions even when there is no overt execution of the action. In this study, we examined whether cognitive processes prior to the physical execution of an action can elicit attentional bias towards irrelevant tasks or dimensions of that action. Participants performed two independent tasks - an action task and a search task-where they were instructed to plan an action and execute the action following the visual search task. We found that the same features of the object were prioritized in the subsequent search task when participants had planned an action response on the object in comparison to when they had not. This effect occurred even when the feature was irrelevant to the tasks or required action. Furthermore, the effect of action planning without physical response was found to be comparable to the effect of physical response. These results suggest that planning of a simple action can induce attentional bias to irrelevant features of objects even without physical action.
\end{abstract}

Keywords Attention $\cdot$ Visual search $\cdot$ Action planning $\cdot$ Action effect

In our daily lives, we interact with many of the objects we see. Interaction with objects can be as simple as pressing a button on a remote control, or as complex as driving a car. To execute such interactions successfully, we often coordinate our physical movements with visual attention; often, our visual perception guides physical action. Many studies have previously shown that actions or action requirements can have an impact on people's cognitive processes, including visual perception (e.g., Neumann \& Prinz, 1990; Schütz-Bosbach \& Prinz, 2007) and memory (Hanning \& Deubel, 2018; Heuer, Crawford, \& Schubö, 2017; Ohl \& Rolfs, 2017).

A recent series of studies found that simple arbitrary action towards an object can induce the processing of features of an acted-on object to be prioritized in a following, unrelated task, and this phenomenon was named "action effect" (Buttaccio \& Hahn, 2011; Suh \& Abrams, 2018; Wang, Sun, Sun, Weidler, \& Abrams, 2017; Weidler \& Abrams, 2014, 2016, 2018). Since the term "action effect" can refer to a broad range of effects that actions have in different areas in psychology, the

Min-Shik Kim

kimm@yonsei.ac.kr

1 Department of Psychology, Yonsei University, 50, Yonsei-ro, Seodaemun-gu, Seoul, Korea "action effect" discussed here will be limited to the following findings only.

In Buttaccio and Hahn's (2011) study, participants first saw a word cue (e.g., a colour or shape name) followed by a coloured stimulus (prime). For the action task, they were instructed to respond to the prime by pressing the space bar if the word and the feature of the prime matched (action), and not to respond if they did not match (no action). Afterwards, participants searched for a tilted line among vertical lines and discriminated the orientation of the target. The target tilted line could appear on a stimulus of the same colour as the prime, or on a stimulus of a different colour with an equal probability. Participants found the target faster when the target appeared in the prime's colour in comparison with when the target appeared in a different colour when they had physically responded to the prime for the action task. However, this validity effect was not observed for the no-action trials where the participants had not physically responded to the prime. In Weidler and Abrams (2014), "GO" or "NO-GO" was used for the word cue, where participants were simply instructed to press the space bar on the prime if the word "GO" was presented. The prioritization of acted-on objects occurred even when participants did not need to evaluate any of the properties of the acted-on object. This prioritization for acted-on objects on visual search has been found in various 
other circumstances, such as in a pop-out search, saccadic responses, semantic associations, and even when the prime was presented outside of visual awareness (Suh \& Abrams, 2018; Wang et al., 2017; Weidler \& Abrams, 2016, 2018).

Findings from the abovementioned studies indicate that a simple action can affect subsequent attentional selection even if properties of the acted-on object are task irrelevant. However, performing an action may involve more processes than a simple physical response. When performing an action, people may also experience covert psychological changes such as changing their internal representations, making an intention for the action, or preparing for an action before they make a behavioural change (Jeannerod, 2001). Neural studies support the idea by showing that voluntary movements are planned and represented in the brain before they are initiated (e.g., Ball et al., 1999; Cunnington, Windischberger, Deecke, \& Moser, 2003). Other studies demonstrated how supplementary motor area (SMA) is involved in the planning and control of the movement (Goldberg, 1985; Roland, Larsen, Lassen, \& Skinhoj, 1980).

Interestingly, action-based change in cognitive processes can occur even before the action is executed or even when there is no overt motor execution of the action. Imagined movements are known to induce neural processes similar to those of actual motor movement (Decety et al., 1994; Grafton, Arbib, Fadiga, \& Rizzolatti, 2004; Jeannerod \& Decety, 1995; Lotze et al., 1999; Stephan et al., 1995). Mental practice or rehearsal has been shown to improve cognitive and motor performance of the related task (Allami, Paulignan, Brovelli, \& Boussaoud, 2008; Driskell, Copper, \& Moran, 1994; Feltz \& Landers, 1983; Wohldmann, Healy, \& Bourne, 2007).

Behavioural studies on action planning also show that planning actions increase the impact of the properties of the stimulus dimension that are relevant or congruent with that type of action. For example, Craighero, Fadiga, Rizzolatti, and Umiltà (1999) showed that preparation of a specifically oriented grasping movement facilitates visual processing of stimuli sharing the same orientation. In the same context, planning a grasping movement facilitated visual processing of size or orientation, and planning a pointing movement facilitated the processing of location or luminance (Bekkering \& Neggers, 2000; Fagioli, Hommel, \& Schubotz, 2007; Wykowska \& Schubö, 2012; Wykowska, Schubö, \& Hommel, 2009). Planning movements can also bias attentional allocation to action-relevant locations (Baldauf \& Deubel, 2008, 2010; Hanning \& Deubel, 2018; Hanning, Jonikaitis, Deubel, \& Szinte, 2016). Rolfs, Jonikaitis, Deubel, and Cavanagh (2011) found presaccadic visual attention shifts to target locations before the eyes started moving. Furthermore, it has been found that action planning not only influences early visual perception but also influences later stages of visual processing (i.e., working memory) on action-relevant feature dimensions (Heuer \& Schubö, 2017). These findings stress that covert cognitive processes associated with action can influence relevant dimensions without overt execution of the action.

Even though previous studies had found that cognitive processes prior to the execution of an action can affect performances, these effects were limited as being specific to the feature dimensions or performances relevant to the action. For example, mentally practicing a certain sequence of typing can increase performance on typing the sequence subsequently (Wohldmann et al., 2007), or if one plans a grasping movement, size information gets prioritized compared with when a pointing movement is planned (Fagioli et al., 2007; Wykowska et al., 2009). Previous planning studies proposed that planning an action facilitates the processing of relevant dimensions for a pragmatic purpose (i.e., it eases the acquisition of information that is useful for the execution of the planned action; Memelink \& Hommel, 2013; Wykowska et al., 2009).

Considering previous studies together, the question of whether planning a simple action can influence irrelevant tasks or dimensions of that action has not been investigated. Will planning an action boost attentional allocation towards features of a task-irrelevant object? Will the processing of a stimulus be facilitated, even when the stimulus does not provide action-relevant information needed to execute that particular action?

To answer these questions, we modified the dual-task paradigm used in action effect studies by Buttaccio and Hahn (2011) and Weidler and Abrams (2014). In contrast to prior studies where participants had always performed the action task on the prime prior to the search task, participants were instructed to only plan an action when the prime appeared and execute the action following the visual search task. In this way, we could investigate whether action-related processes other than the execution of an action can also affect how attention is distributed.

Most importantly, the purpose of this study was to examine whether planning this simple action can have an influence on tasks or feature dimensions irrelevant to the planned action. If action planning can affect deployment of attention to the action-irrelevant properties, facilitation of processing feature information that does not give benefits for the execution of that type of action would be exhibited.

We hypothesized that planning of an action upon the prime will be sufficient to induce prioritization of attention to the prime. Consequently, a larger validity effect on actionplanning trials will be produced compared with no-actionplanning trials, even though both action-planning and noaction-planning trials did not execute any physical movement. However, if physical movement is a prerequisite for an action to induce the attentional prioritization on task-irrelevant features of the objects, we would not observe a larger validity effect on action-planning trials. 


\section{Experiment 1}

In Experiment 1, we examined whether planning a simple action can bias attention towards planned-upon features of objects in the task irrelevant to the planned action. We used a modified version of the paradigm used by Buttaccio and Hahn (2011). Participants were presented with a colour word and then the prime. Participants were instructed to judge whether the colour word and the prime colour matched or mismatched for the later action task. Afterwards, participants performed a visual search task, and, lastly, conducted an action task where they executed the planned action. We expected the processing of the prime to be enhanced in the search task when participants planned for an action on the prime relative to when they did not.

\section{Method}

Participants Forty-eight undergraduate students from Yonsei University (26 females) participated for course credit. All participants reported normal or corrected-to-normal vision. All procedures were approved by the Institutional Review Board of Yonsei University. Participants were randomly assigned to either match-action or mismatch-action group. The number of participants was determined through power analysis using effect sizes obtained from a prior study (Weidler \& Abrams, 2014). ${ }^{1}$

Two participants were removed from the analysis for correctly responding to both the action task and the search task in less than $80 \%$ of all trials.

Stimuli and apparatus Stimuli were presented on a 24-inch LED monitor with a $60-\mathrm{Hz}$ refresh rate and $1,920 \times 1,080$ resolution. The experiment was conducted on an Intel QuadCore level computer and operated via MATLAB equipped with Psychophysics Toolbox Extension 3.0 (Brainard, 1997). Distance from the monitor to participants' eyes was about $57 \mathrm{~cm}$. Participants' heads were stabilized using chin and forehead rests.

All stimuli were presented with a black $\left(0 \mathrm{~cd} / \mathrm{m}^{2}\right)$ background. Colour words ("red," "yellow," "green," "blue," "purple," and "grey") appeared in Korean, in a white colour with a font size of 30 pixels at the centre of the screen. Prime and search

\footnotetext{
${ }^{1}$ Weidler and Abrams's (2014) Experiment 2 was used for determining the number of participants because one of the conditions of that experiment had an experimental design most similar to our study, except that we changed "action" to "action planning." Given two-tailed tests and an alpha level of .05, the minimum sample size required to achieve a $95 \%$ or greater power to detect the action effect (Weidler \& Abrams, 2014, Experiment 2, Action $\times$ Validity interaction in no-offset condition; $n_{p}^{2}=.57$ ) on reaction time was 14 . Since a between-participants variable was added for counterbalancing, and the effect of planning without physical action was examined unlike in the prior study, more participants were thought to be needed than the acquired minimum sample size.
}

stimuli were each in circles of $135 \times 135$ pixels $\left(4^{\circ} \times 4^{\circ}\right.$ of visual angle), in one of six colours - red, yellow, green, blue, purple, and grey (controlled in luminance, $70 \mathrm{~cd} / \mathrm{m}^{2}$, and saturation). In the visual search array, two search stimuli appeared at two of five locations of equal distance and angle from the centre of the screen and each stimulus contained a black line. One stimulus contained a target line tilted to either the left or right $\left(31.53^{\circ}\right)$ and the other circle contained a vertical distractor line. In the action task, a white circle ring of $135 \times 135$ pixels $\left(4^{\circ} \times 4^{\circ}\right.$ of visual angle) in the centre was used as a stimulus.

Procedure The experimental stimuli and procedure are shown in Fig. 1. Each trial began with a fixation cross presented for $500 \mathrm{~ms}$. Then, one of the colour words was presented for 500 ms. After a 130-ms fixation, a coloured circle appeared as a prime for $750 \mathrm{~ms}$. After another fixation for $500 \mathrm{~ms}$, participants were presented with a visual search array that contained two different coloured circles. One circle was in the same colour as the prime and the other was in a different colour, randomly chosen out of the five remaining colours. One of the circles contained a vertical line as the distractor, and the other contained a tilted line as the target. Participants had to report the orientation of the target line as tilting left or right by using the left or right arrow keys. On valid trials, the target was embedded in the circle with the same colour as the prime; on invalid trials, the target was embedded in the circle with a different colour than the prime. The search display remained on screen for $1,500 \mathrm{~ms}$. After a 500-ms delay, a white ring appeared for 1,500 ms or until there was a response. To control the effect of the colour word-prime match, the mapping between the colour word-prime match and action was counterbalanced across participants. The "match-action group" was instructed to press the space bar (go) if the colour word and the prime colour had matched, and not to press the space bar (no-go) if the colour word and the prime colour had not matched. The "mismatch-action group" was instructed to press the space bar if the colour word and the prime colour had not matched. Auditory feedback was provided for incorrect or slow responses both for the action task and search task.

Participants performed 24 practice trials and six blocks of 24 trials (total of 144 trials), half of which were actionplanning (go) trials and the other half were no-actionplanning (no-go) trials. Each of the six colours were presented equally as a prime. The location of the two circles (among the five predetermined locations), the second colour in the search display, and the target orientation were chosen at random. Features of the prime did not benefit the search task since there were equal numbers of valid and invalid trials.

\section{Results}

Only the trials in which participants answered both the action task and the search task correctly were included in the 


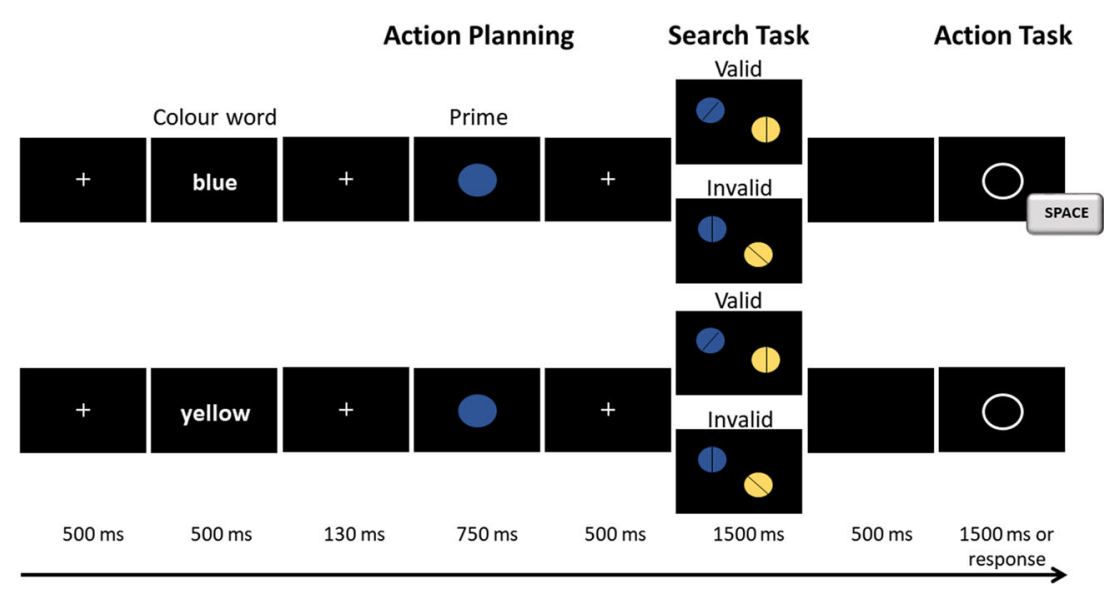

Fig. 1 Example of stimuli and procedures in Experiment 1. First, a colour word (in Korean) and then a prime was presented. Participants had to evaluate whether the colour word and the colour of the prime matched. Afterwards, a search array was presented where participants had to respond to the orientation of a tilted line. After the search task, the match-action group were instructed to press the space bar (go) if the colour word and the prime colour had matched and not to press anything (no-go) if the colour word and the prime colour had not matched. The instruction for the action task was reversed for the mismatch-action group

Since the results from Experiment 1 were confounded with the effect of the word-prime match, we conducted an additional analysis as Buttaccio and Hahn (2011) had performed when they compared "match action" trials with "match no-action" trials to see the unique effect of action planning. We compared word-prime match trials of each group where the match-action group planned an action ("match go") and the mismatchaction group did not plan an action ("match no-go"). We chose go/no-go (go, no-go) as a between-subjects variable, and prime validity (invalid, valid) as a within-subjects variable (see Fig. 2c).

The main effect of prime validity was significant. Participants responded faster when the prime was valid as opposed to being invalid, $F(1,44)=34.45, p<.001, \eta_{p}^{2}=$ .44. There was no main effect of go/no-go, $F(1,44)=.35, p=$ $.558, \eta_{p}^{2}=.01$. There was a significant interaction between go/ no-go and prime validity, with a larger validity effect on go trials than on no-go trials, $F(1,44)=9.43, p=.004, \eta_{p}^{2}=.18$.

\section{Discussion}

In Experiment 1, we aimed to investigate whether "planning of an action" alone without physical execution of the action can bias attention towards planned-upon features in the subsequent task. Participants planned an action or did not do so according to the match of the colour word and the prime and performed the action task after the visual search. The results showed that the validity effect of the prime was larger in the subsequent search task when participants planned an action compared with when they did not, indicating that planning an action was sufficient for altering the allocation of attention in the search task. 
$\mathbf{a}$

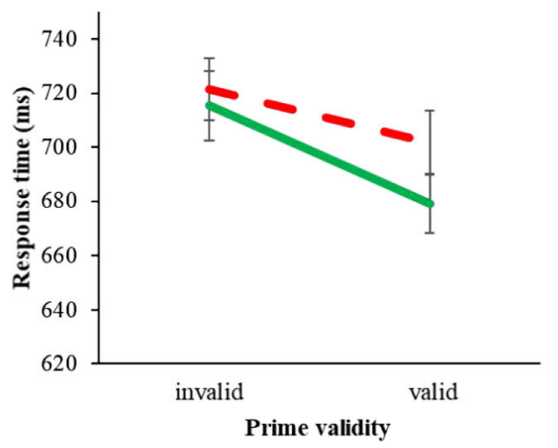

b

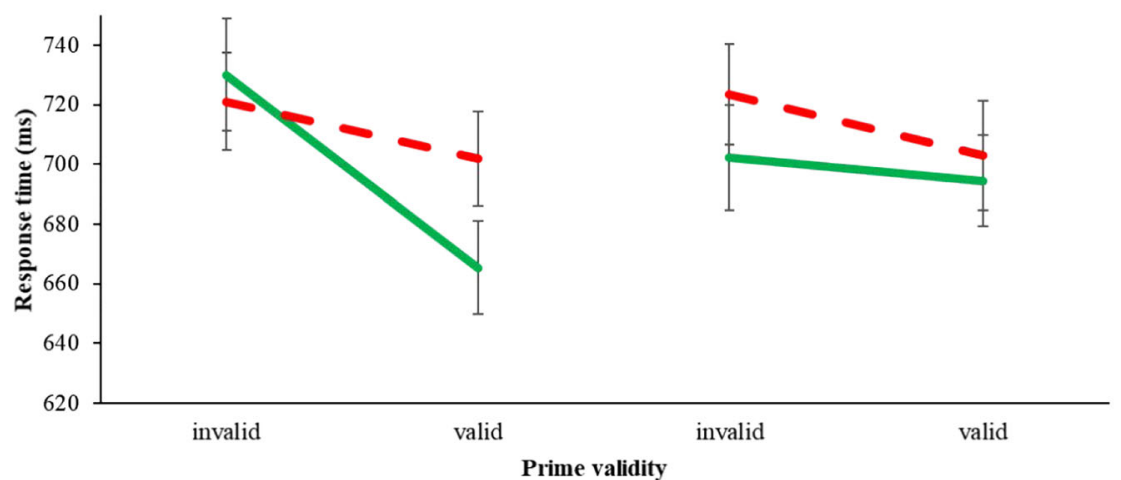

Word-Prime Match condition

Mismatch-action group

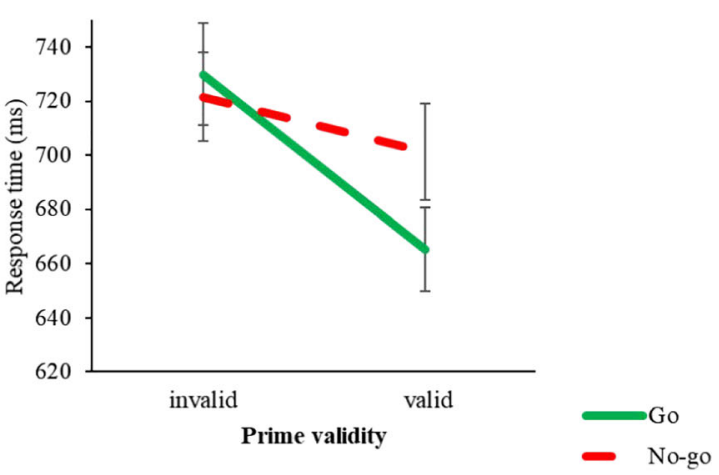

no-go trials when the word matched with the prime. All error bars depict standard errors of the means

controlled in their study, it is possible that the action effects were confounded with the cue-prime match effect.

Even though we counterbalanced the effect of word-prime match, one might still argue that the larger validity effect in action trials found here is also caused by the effect of wordprime match and not by the action planning. However, for the mismatch-action group, we could not find a facilitation effect from a word-prime match in a no-action condition. More importantly, in the additional analysis where we compared go and no-go trials when the word-prime had matched for each group, we were able to find a unique effect of action planning in the validity effect. These results support that both the effect of the attentional set and action planning influence attentional selection, and they are closely intertwined. However, further research appears to be needed to determine how they interact with each other.

Although the colour dimension was irrelevant to the search task in Experiment 1, it is difficult to say that colour dimension was completely irrelevant for participants since the action task was about evaluating colours. The attentional set hypothesis proposes that features of a planned-upon prime might capture more attention only when participants are attuned to that particular feature dimension (Folk, Remington, \& 
Johnston, 1992). Similarly, one could suggest that the effect of action planning towards the prime occurs only when participants evaluate any of the properties of the prime. To address these issues, we conducted Experiment 2 to investigate whether the mere spatiotemporal association of the prime and action planning can cause the prioritization of the prime.

\section{Experiment 2}

Experiment 2 was performed to further investigate the following. First, since the effect of action planning and word-prime match were confounded in Experiment 1, we aimed to examine the effect of action planning more clearly by excluding the effect of word-prime match. In addition, we tested whether the planning can modify attention even when the feature of the prime is irrelevant to both the action task and the search task and even when participants do not need to process any of the features of the prime. If selective attention can be altered by mere spatiotemporal overlap between the prime and the action planning, we expected to observe a larger validity effect in action-planning trials.

Even though participants were not required to remember the prime but only required to remember whether to perform action or not in the current study, it is possible that people remembered the prime better in actionplanning trials relative to no-action-planning trials, which led to a larger validity effect in the search task. It is well known that contents of working memory can affect the allocation of visual attention (e.g., Han \& Kim, 2009; Olivers, Peters, Houtkamp, \& Roelfsema, 2011; Soto, Heinke, Humphreys, \& Blanco, 2005). To examine whether the larger validity effect in action-planning trials is due to better working memory of the prime, an extra trial was added at the end of the experiment, with a surprise question probing the prime's colour. If the larger validity effect is caused by a better working memory, we expected to find better memory performance for the colour of the prime in action-planning trials compared with no-action-planning trials. However, if action planning does not induce better working memory for the prime, we expected to not find any difference in memory performance between action-planning trials and no-actionplanning trials.

Furthermore, we aimed to examine whether planning yields effects as strong as actual action and a physical response does. Executing a physical response might give an additive effect on allocation of attention, yielding a larger validity effect when physically acted compared with when only planned. Half of the participants were instructed to perform the action task when the prime appeared as in original action effect studies (e.g., Buttaccio \& Hahn, 2011; Weidler \&
Abrams, 2014), and we compared the effect of actual action with the effect of action planning.

\section{Method}

Participants Forty-eight undergraduate students from Yonsei University (30 females) participated for course credit or monetary compensation (\$5). All participants reported normal or corrected-to-normal vision. Participants were randomly assigned to either the action planning group or the actual action group. The number of participants was determined through power analysis using effect sizes obtained from a prior study (Weidler \& Abrams, 2014). ${ }^{2}$

Stimuli and procedure The procedure for the action planning group is shown in Fig. 3. The only part that was different from the previous experiment was the action task. First, a prime was presented for $750 \mathrm{~ms}$. While the prime was being presented, a stimulus in a T-shape or an L-shape appeared on the prime for $200 \mathrm{~ms}$. Participants were randomly assigned to T or L for the target in action task. Participants were instructed to press the space bar (go) after the search task if the assigned target stimulus had appeared on the prime. The procedure was identical for the actual action group, except that participants performed the action task before the search task while the prime was being presented.

After the test trials, participants were given a single extra trial with surprise questions. Participants were presented with the same procedure with the test trials until the search task. After the search task, however, instead of the action task, two surprise questions were presented sequentially. Participants were first asked to report the colour of the prime among the six colours, and then to report their confidence.

\section{Results}

The trial exclusions were removed in the same way as in Experiment 1, which resulted a removal of about $7.75 \%$ of the data. The accuracy for the action task was $96.66 \%(S D=$ $3.44 \%)$ and the search task was $97.89 \%(S D=2.11 \%)$.

Figure 4 shows mean RTs for the search task. A three-way ANOVA with task type (action planning group/actual action group) as a between-subject variable and go/no-go (go/no-go) and prime validity (valid/invalid) as the two within-subject variables was conducted. There was a main effect of prime validity, with faster responses to valid trials compared with

\footnotetext{
${ }^{2}$ Weidler and Abrams's (2014) Experiment 4 was used for determining the number of participants for the same reason as in Experiment 1. Given twotailed tests and an alpha level of .05, the minimum sample size required to achieve a 95\% or greater power to detect the action effect (Weidler \& Abrams, 2014 , Experiment $4 ; n_{p}^{2}=.46$ ) on reaction time was 19 . Since a betweenparticipants variable was added, more participants were thought to be needed than the acquired minimum sample size.
} 


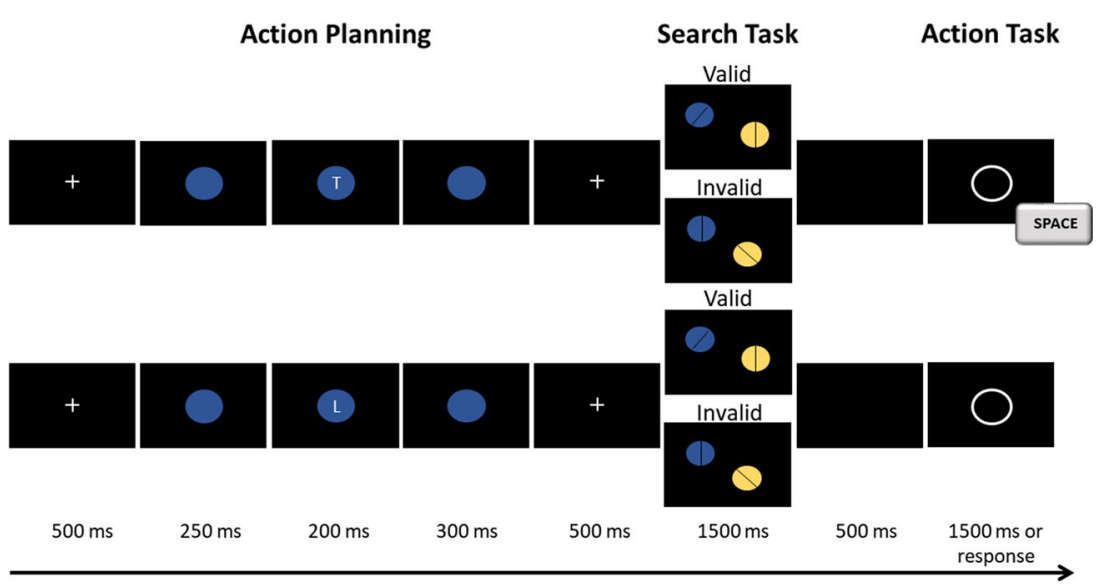

Fig. 3 Example of stimuli and procedures in Experiment 2. First, a prime was presented. While the prime was presenting, a stimulus in a T-shape or L-shape appeared and disappeared briefly. For the acutal action group, participants were instructed to press the space bar (go) right after if $\mathrm{T}$ (or

invalid trials, $F(1,46)=28.71, p<.001, \eta_{p}^{2}=.38$. No main effect of go/no-go was observed, $F(1,46)=2.77, p=.103, \eta_{p}^{2}$ $=.06$, nor the main effect of task type, $F(1,46)=1.52, p=$ .224, $\eta_{p}^{2}=0.03$. Critically, we found an interaction between go/no-go and prime validity, $F(1,46)=13.55, p<.001, \eta_{p}^{2}=$ .23. The interaction between go/no-go and prime validity was statistically significant both for the action planning group, $F(1,23)=4.75, p=.040, \eta_{p}^{2}=0.17$, and the actual action group, $F(1,23)=8.90, p=.007, \eta_{p}^{2}=0.28$. None of the other interactions were significant $\left(F<1, p>.40, \eta_{p}^{2}<.02\right.$ for all).

To assess whether participants remembered the colour of the prime better in go trials than in no-go trials, we compared the number of participants who correctly recalled the colour of the prime in the surprise memory trial. A chi-squared analysis showed that there was no significant difference in the number of correctly recalled participants between different go/no-go conditions, $\chi^{2}(1, N=48)<1, p=.768$. No difference was found for both the action planning group ( $\mathrm{go}=3$, no go $=3$ ),

\section{Action planning group}

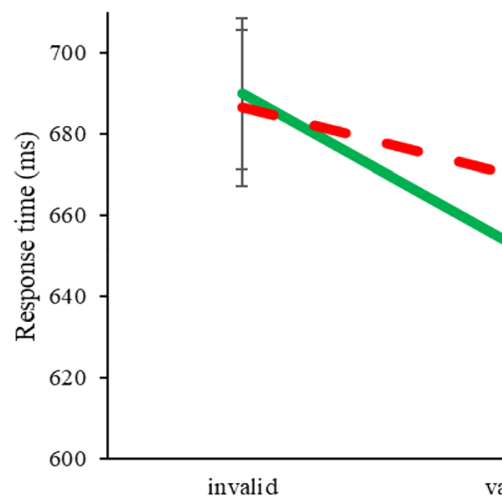

L; randomly counterbalanced) appeared on the prime. For the action planning group, participants were instructed to press the space bar after performing a visual search task. The figure depicts the procedure for the action planning group for whom $\mathrm{T}$ was the target

$\chi^{2}(1)<1, p=1$, and the actual action group ( $\mathrm{go}=6$, no go $=$ $7), \chi^{2}(1)<1, p=.682$. However, a significant difference was found between the task type, $\chi^{2}(1)=4.27, p=.039$. The action planning group had six participants who were correct in their recall out of total 24 participants, and the actual action group had 13 such participants out of a total 24 participants. Chi-square tests showed that the number of correctly recalling participants was not significantly higher than chance for the action planning group, $\chi^{2}(1)=1.20, p=.273$., but it was higher than chance for the actual action group, $\chi^{2}(1)=$ $24.30, p<.001$.

\section{Discussion}

In Experiment 2, we controlled for the effect of word-prime match and were still able to observe the effect of action planning. The response time difference between invalid and valid trials was larger when participants planned an action than when they did not. Furthermore, the colour of the prime

\section{Actual action group}

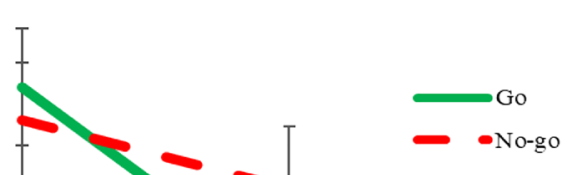

Prime validity

Fig. 4 RTs in visual search task for each condition in Experiment 2. Error bars depict standard errors of the means 
influenced participants' search performance even though neither the colour dimension nor the prime itself was relevant for any of the tasks that the participants performed. This finding indicates that processing of the object is not needed, and simple spatiotemporal overlap of the prime and action planning is sufficient to induce the effect of action planning.

Furthermore, we were able to replicate the action effect shown in previous studies (e.g., Buttaccio \& Hahn, 2011; Weidler \& Abrams, 2014). Participants showed a larger validity effect when they pressed the space bar on the prime compared with when they did not. Even though the validity effect caused by actual action was slightly larger ( $49 \mathrm{~ms})$ than the validity effect caused by planning $(37 \mathrm{~ms})$, the difference was not statistically significant. This result indicates that mental presentation of action without physical response can influence attention allocation as strongly as actual action can.

The results of the surprise memory task of prime's colour revealed no difference in performance between go and no-go trials. Based on the results, it seems unlikely that better working memory in go trials elicited the larger validity effect in the search task. No memory performance difference between go and no-go trials in the actual action group further implies that the action effect constantly shown in previous studies (e.g., Buttaccio \& Hahn, 2011; Weidler \& Abrams, 2014) may not be due to differences in working memory, also.

Conversely, there was a significant difference in memory performance according to the task type. Participants who were asked to perform the actual action on the prime were better at remembering the colour of the prime than the participants who were asked to plan. Although the task type did not influence performance in the search task, it is possible that the different demands of the tasks induced different cognitive processes. However, these results should be interpreted with caution since it was a one-trial memory test. A one-trial surprise memory test at the end of the experiment was used because participants may memorize the colour of the prime intentionally after the first surprise memory test. Further evidence with novel methodologies will be needed to rule out the possibility that the effects are caused by working memory.

\section{General discussion}

The goal of the present study was to investigate whether planning of a simple action alone can bias attention allocation towards planned-upon objects on irrelevant tasks or on irrelevant feature dimensions of that action. In the present study, participants planned to press the space bar according to the task and performed the action after a visual search task. In Experiment 1, participants planned the action by the match or mismatch of the colour word and the prime. A larger validity effect of the prime in the search task was observed when participants planned for an action than when participants did not, indicating that the action planning induced increased allocation of attention on the prime. In Experiment 2, participants planned the action by an irrelevant letter stimulus appearing on the prime. The processing of the colour of the prime was facilitated by action planning even when the colour dimension or the prime was irrelevant to participants in any of the tasks. Additionally, comparison with the effect of actual action showed that the effect of action planning is comparable to the effect of actual action, and neither the effect of the action planning nor the action was caused by a better working memory of the prime.

Previous studies have shown that the mental representation of a specific action can enhance performance of that action (Allami et al., 2008; Driskell et al., 1994; Feltz \& Landers, 1983; Wohldmann et al., 2007). Other studies have shown that planning specific actions towards objects enhances the salience of the relevant feature dimensions in executing those actions (Bekkering \& Neggers, 2000; Craighero et al., 1999; Fagioli et al., 2007; Symes, Tucker, Ellis, Vainio, \& Ottoboni, 2008; Wykowska et al., 2009). In this account, when the required action needs specific featural information for the execution, planning of that action facilitates the featural information that provides benefits for the execution of that particular type of action. However, an arbitrary action (i.e., pressing the space bar) was used for an action. Compared with the actions used in previous studies, such as pointing or grasping, pressing the space bar needs less coordination with objects. Therefore, it does not have distinctive feature dimensions needed to aid the execution of the action. Furthermore, colour dimension does not provide useful information for executing the particular action "pressing the space bar." Still, we were able to determine that planning a simple action alone is sufficient to affect attention in a subsequent search task, prioritizing the planned-upon features of the object that were not relevant to the search task. Particularly in Experiment 2, the stimulus presented as a prime was not relevant to any of the tasks that participants performed. In other words, with just a mere spatiotemporal overlap of the presentation of the object and participants deciding whether to act on the action task, the task-irrelevant features of the object were able to influence how attention was allocated in a subsequent task.

How, then, could planning an action induce biased attention towards a task-irrelevant object or feature dimension? Weidler and Abrams (2014) mentioned that the effect of action in their studies might be showing the effect of selection history. Previous studies have found that the recent history of attentional deployments can induce prolonged selection biases even when there are no top-down explicit goals of the observer or bottom-up physical salience of the stimulus (Awh, Belopolsky, \& Theeuwes, 2012; Theeuwes, 2019). One of the phenomena of selection history is short-term intertrial priming, where a stimulus that was attended in past trials is selected more efficiently in the next trial (e.g., Kristjánsson \& 
Campana, 2010; Maljkovic \& Nakayama, 1994). Even though action planning could have worked as "selection" in a similar way, the difference from previous studies is that only a single stimulus was presented during action planning without any other stimuli competing for attentional selection. We were able to determine that planning can enhance the processing of a stimulus even when competition for attentional selection is not present.

Another potential mechanism similar to the current study is the "attentional boost effect" (Makovski, Jiang, \& Swallow, 2013; Swallow \& Jiang, 2010, 2011; Swallow, Makovski, \& Jiang, 2012). In their paradigms, participants were presented with a series of background images while they performed a target-detection task that was irrelevant to the presented images. They found that images appearing with targets of a detection task were better recognized or remembered than were images appearing with nontargets. The authors indicated that detecting a target in one task may open an attentional "gate," enhancing the processing of other information presented at the time of the target. It is possible that the prime in our study might have gained facilitation in a similar way as attentional boost effect. Although participants did not overtly respond to the prime, planning an action may have worked similarly to finding a target, therefore the prime gained more attentional deployment. Makovski et al. (2013) also observed better memory for internally counted images than for those that were not counted internally. However, while previous attentional boost effect studies observed enhancement in memory or in working memory, we could not find the evidence for better memory for the prime in actionplanning trials compared with no-action-planning trials. Even though Swallow et al. (2012) suggested that attentional boost effect might occur in early encoding stage, further investigation seems to be needed as to whether the current boost effect in attentional capture shares the same mechanisms as attentional boost effect in memory.

According to the working memory account, better working memory for the colour of the prime in action-planning trials compared with no-action-planning trials may lead to a larger validity effect in action-planning trials (e.g., Han \& Kim, 2009; Olivers et al., 2011; Soto et al., 2005). Inconsistent with the working memory account, we could not find a statistical difference in surprise memory performances between go and no-go trials in Experiment 2. This suggests that the larger validity effect in action-planning trials may not be elicited by a difference in the contents of working memory. However, the interpretation on the memory task should not be made conclusively in this paper due to its limited experimental design and that it was only a one-trial task. Further evidence is needed through novel experimental designs about whether participants memorize the colour of the prime even if they are not asked to do so.
Lastly, although we did not observe an additive effect of physical movement compared with only planning an action in response times, the memory performance on the colour of the prime was better for the participants who performed the actual action on the prime than the participants who were only involved in action planning on the prime. It is possible that the different methodology used for each condition may have caused this difference such as a heightened alertness for the actual action group since they had to respond immediately after the presentation of the prime within a limited time (500 $\mathrm{ms})$ compared with the action planning group $(1,500 \mathrm{~ms})$. On the other hand, it may imply that execution of an action with physical movement involves different cognitive processes compared with planning. Previous researchers have found that even though imagined movement and real movement share similar neural activations, some areas are differentially activated (Gerardin, 2000; Grafton et al., 2004; Lotze et al., 1999; Stephan et al., 1995). Otherwise, it is also possible that physical movement becomes more important when a task requires more precise movement. A meta-analysis study found that mental practice was effective in improving performances, but it was more effective in cognitive tasks than in physical tasks (Driskell et al., 1994). It will be interesting to investigate further about the role of physical movement in action and the differences in action planning and execution in future research.

In summary, the effect of action on the deployment of attention in subsequent unrelated tasks occurred without the execution of physical action. Importantly, the feature of the object that was spatiotemporally associated with action planning was prioritized even when the feature was irrelevant to the required action or tasks.

Open practices statement The data for all experiments are available at https://osf.io/t4zpd/. None of the experiments was preregistered.

Acknowledgements This work was supported by the Ministry of Education of the Republic of Korea and the National Research Foundation of Korea (NRF-2018S1A5A2A01032827) and by the Yonsei University Research Fund of 2018 (2018-22-0102).

\section{References}

Allami, N., Paulignan, Y., Brovelli, A., \& Boussaoud, D. (2008). Visuomotor learning with combination of different rates of motor imagery and physical practice. Experimental Brain Research, 184(1), 105113. https://doi.org/10.1007/s00221-007-1086-x

Awh, E., Belopolsky, A. V., \& Theeuwes, J. (2012). Top-down versus bottom-up attentional control: A failed theoretical dichotomy. Trends in Cognitive Sciences, 16(8), 437-443. https://doi.org/10. 1016/j.tics.2012.06.010 
Baldauf, D., \& Deubel, H. (2008). Visual attention during the preparation of bimanual movements. Vision Research, 48(4), 549-563. https:// doi.org/10.1016/j.visres.2007.11.023

Baldauf, D., \& Deubel, H. (2010). Attentional landscapes in reaching and grasping. Vision Research, 50(11), 999-1013. https://doi.org/10. 1016/j.visres.2010.02.008

Ball, T., Schreiber, A., Feige, B., Wagner, M., Lücking, C., \& KristevaFeige, R. (1999). The role of higher-order motor areas in voluntary movement as revealed by high-resolution EEG and fMRI. NeuroImage, 10(6), 682-694. https://doi.org/10.1006/nimg.1999. 0507

Bekkering, H., \& Neggers, S. F. W. (2000). Visual search is modulated by action intentions. Psychological Science, 13(4), 370-374. https:// doi.org/10.1111/j.0956-7976.2002.00466.x

Brainard, D. H. (1997). The psychophysics toolbox. Spatial Vision, 10(4), 433-436. https://doi.org/10.1163/156856897X00357

Buttaccio, D. R., \& Hahn, S. (2011). The influence of action on visual search: Behavioral response toward stimuli modifies the selection process. Attention, Perception, \& Psychophysics, 73(5), 1453-1466. https://doi.org/10.3758/s13414-011-0121-y

Craighero, L., Fadiga, L., Rizzolatti, G., \& Umiltà, C. (1999). Action for perception: A motor-visual attentional effect. Journal of Experimental Psychology: Human Perception and Performance, 25(6), 1673-1692. https://doi.org/10.1037/0096-1523.25.6.1673

Cunnington, R., Windischberger, C., Deecke, L., \& Moser, E. (2003). The preparation and readiness for voluntary movement: A high-field event-related fMRI study of the Bereitschafts-BOLD response. NeuroImage, 20(1), 404-412. https://doi.org/10.1016/S10538119(03)00291-X

Decety, J., Perani, D., Jeannerod, M., Bettinardi, V., Tadary, B., Woods, R., ... Fazio, F. (1994). Mapping motor representations with positron emission tomography. Nature, 371(6498), 600-602. https://doi. org/10.1038/371600a 0

Driskell, J. E., Copper, C., \& Moran, A. (1994). Does Mental Practice Enhance Performance? Journal of Applied Psychology, 79(4), 481492. https://doi.org/10.1037/0021-9010.79.4.481

Fagioli, S., Hommel, B., \& Schubotz, R. I. (2007). Intentional control of attention: Action planning primes action-related stimulus dimensions. Psychological Research, 71(1), 22-29. https://doi.org/10. 1007/s00426-005-0033-3

Feltz, D. L., \& Landers, D. M. (1983). The effects of mental practice on motor skill learning and performance: A meta-analysis. Journal of Sport Psychology, 5(1), 25-57.

Folk, C. L., Remington, R. W., \& Johnston, J. C. (1992). Involuntary covert orienting is contingent on attentional control settings. Journal of Experimental Psychology: Human Perception and Performance, 18(4), 1030-1044. https://doi.org/10.1037/0096-1523.18.4.1030

Gerardin, E. (2000). Partially overlapping neural networks for real and imagined hand movements. Cerebral Cortex, 10(11), 1093-1104. https://doi.org/10.1093/cercor/10.11.1093

Goldberg, G. (1985). Supplementary motor area structure and function: Review and hypotheses. Behavioral and Brain Sciences, 8(1985), 567-616. https://doi.org/10.1017/S0140525X00045167

Grafton, S., Arbib, M., Fadiga, L., \& Rizzolatti, G. (2004). Localization of grasp representations in humans by positron emission tomography. Experimental Brain Research, 112(1), 103-111. https://doi. org $/ 10.1007 / \mathrm{bf00227183}$

Han, S. W., \& Kim, M. S. (2009). Do the contents of working memory capture attention? Yes, but cognitive control matters. Journal of Experimental Psychology: Human Perception and Performance, 35(5), 1292-1302. https://doi.org/10.1037/a0016452

Hanning, N. M., \& Deubel, H. (2018). Independent effects of eye and hand movements on visual working memory. Frontiers in Systems Neuroscience, 12(August), 1-5. https://doi.org/10.3389/fnsys.2018. 00037
Hanning, N. M., Jonikaitis, D., Deubel, H., \& Szinte, M. (2016). Oculomotor selection underlies feature retention in visual working memory. Journal of Neurophysiology, 115(2), 1071-1076. https:// doi.org/10.1152/jn.00927.2015

Heuer, A., \& Schubö, A. (2017). Selective weighting of action-related feature dimensions in visual working memory. Psychonomic Bulletin \& Review, 24(4), 1129-1134. https://doi.org/10.3758/ s13423-016-1209-0

Heuer, A., Crawford, J. D., \& Schubö, A. (2017). Action relevance induces an attentional weighting of representations in visual working memory. Memory \& Cognition, 45(3), 413-427. https://doi.org/10. 3758/s13421-016-0670-3

Jeannerod, M. (2001). Neural simulation of action: A unifying mechanism for motor cognition. NeuroImage, 14(1), 103-109. https://doi. org/10.1006/nimg.2001.0832

Jeannerod, M., \& Decety, J. (1995). Mental motor imagery: A window into the representational stages of action. Current Opinion in Neurobiology, 5(6), 727-732. https://doi.org/10.1016/09594388(95)80099-9

Kristjánsson, Á., \& Campana, G. (2010). Where perception meets memory: A review of repetition priming in visual search tasks. Attention, Perception, \& Psychophysics, 72(1), 5-18. https://doi.org/10.3758/ APP.72.1.5

Lotze, M., Montoya, P., Erb, M., Hülsmann, E., Flor, H., Klose, U., ... Grodd, W. (1999). Activation of cortical and cerebellar motor areas during executed and imagined hand movements: An fMRI study. Journal of Cognitive Neuroscience, 11(5), 491-501. https://doi.org/ 10.1162/089892999563553

Makovski, T., Jiang, Y. V., \& Swallow, K. M. (2013). How do observer's responses affect visual long-term memory? Journal of Experimental Psychology: Learning Memory and Cognition, 39(4), 1097-1105. https://doi.org/10.1037/a0030908

Maljkovic, V., \& Nakayama, K. (1994). Priming of pop-out: I. Role of features. Memory \& Cognition, 22(6), 657-672. https://doi.org/10. 3758/BF03209251

Memelink, J., \& Hommel, B. (2013). Intentional weighting: A basic principle in cognitive control. Psychological Research, 77(3), 249-259. https://doi.org/10.1007/s00426-012-0435-y

Neumann, O., \& Prinz, W. (1990). Prologue: Historical approaches to perception and action. In O. Neumann \& W. Prinz (Eds.), Relationships between perception and action (pp. 5-19). Berlin, Heidelberg: Springer.

Ohl, S., \& Rolfs, M. (2017). Saccadic eye movements impose a natural bottleneck on visual short-term memory. Journal of Experimental Psychology: Learning Memory and Cognition, 43(5), 736-748. https://doi.org/10.1037/xlm0000338

Olivers, C. N. L., Peters, J., Houtkamp, R., \& Roelfsema, P. R. (2011). Different states in visual working memory: When it guides attention and when it does not. Trends in Cognitive Sciences, 15(7), 327-334. https://doi.org/10.1016/j.tics.2011.05.004

Robinson, M. M., Clevenger, J., \& Irwin, D. E. (2018). The action is in the task set, not in the action. Cognitive Psychology, 100(November 2017), 17-42. https://doi.org/10.1016/j.cogpsych.2017.11.005

Roland, P. E., Larsen, B., Lassen, N. A., \& Skinhoj, E. (1980). Supplementary motor area and other cortical areas in organization of voluntary movements in man. Journal of Neurophysiology, 43(1), 118-136. https://doi.org/10.1152/jn.1980.43.1.118

Rolfs, M., Jonikaitis, D., Deubel, H., \& Cavanagh, P. (2011). Predictive remapping of attention across eye movements. Nature Neuroscience, 14(2), 252-258. https://doi.org/10.1038/nn.2711

Schütz-Bosbach, S., \& Prinz, W. (2007). Perceptual resonance: Actioninduced modulation of perception. Trends in Cognitive Sciences, 11(8), 349-355. https://doi.org/10.1016/j.tics.2007.06.005

Soto, D., Heinke, D., Humphreys, G. W., \& Blanco, M. J. (2005). Early, involuntary top-down guidance of attention from working memory. Journal of Experimental Psychology: Human Perception and 
Performance, 31(2), 248-261. https://doi.org/10.1037/0096-1523. 31.2.248

Stephan, K. M., Fink, G. R., Passingham, R. E., Silbersweig, D., Ceballos-Baumann, A. O., Frith, C. D., \& Frackowiak, R. S. (1995). Functional anatomy of the mental representation of upper extremity movements in healthy subjects. Journal of Neurophysiology, 73(1), 373-386. https://doi.org/10.1152/jn.1995. 73.1 .373

Suh, J., \& Abrams, R. A. (2018). Action influences unconscious visual processing. Attention, Perception, \& Psychophysics, 80(6), 15991608. https://doi.org/10.3758/s13414-018-1509-8

Swallow, K. M., \& Jiang, Y. V. (2010). The attentional boost effect: Transient increases in attention to one task enhance performance in a second task. Cognition, 115(1), 118-132. https://doi.org/10. 1016/j.cognition.2009.12.003

Swallow, K. M., \& Jiang, Y. V. (2011). The role of timing in the attentional boost effect. Attention, Perception, \& Psychophysics, 73(2), 389-404. https://doi.org/10.3758/s13414-010-0045-y

Swallow, K. M., Makovski, T., \& Jiang, Y. V. (2012). Selection of events in time enhances activity throughout early visual cortex. Journal of Neurophysiology, 108(12), 3239-3252. https://doi.org/10.1152/jn. 00472.2012

Symes, E., Tucker, M., Ellis, R., Vainio, L., \& Ottoboni, G. (2008). Grasp preparation improves change detection for congruent objects. Journal of Experimental Psychology: Human Perception and Performance, 34(4), 854-871. https://doi.org/10.1037/0096-1523. 34.4.854

Theeuwes, J. (2019). Goal-driven, stimulus-driven, and history-driven selection. Current Opinion in Psychology, 29(October), 97-101. https://doi.org/10.1016/j.copsyc.2018.12.024

Wang, F., Sun, J., Sun, P., Weidler, B. J., \& Abrams, R. A. (2017). Influence of simple action on subsequent manual and ocular responses. Attention, Perception, \& Psychophysics, 79(2), 389395. https://doi.org/10.3758/s13414-017-1280-2

Weidler, B. J., \& Abrams, R. A. (2014). Decomposing the action effect: How simple actions affect subsequent perception. Attention, Perception, \& Psychophysics, 76(4), 1242-1252. https://doi.org/ 10.3758/s13414-014-0652-0

Weidler, B. J., \& Abrams, R. A. (2016). Simple actions influence pop-out search. Visual Cognition, 24(7/8), 392-405. https://doi.org/10.1080/ 13506285.2017.1289996

Weidler, B. J., \& Abrams, R. A. (2018). Simple actions activate semantic associations. Psychonomic Bulletin \& Review, 25(4), 1500-1506. https://doi.org/10.3758/s13423-017-1415-4

Weidler, B. J., Abrams, R. A., \& Pratt, J. (2018). Is deciding to act or executing the action critical for the action effect? Journal of Vision, 18(10), 843. https://doi.org/10.1167/18.10.843

Wohldmann, E. L., Healy, A. F., \& Bourne, L. E. (2007). Pushing the limits of imagination: Mental practice for learning sequences. Journal of Experimental Psychology: Learning, Memory, and Cognition, 33(1), 254-261. https://doi.org/10.1037/0278-7393.33. 1.254

Wykowska, A., \& Schubö, A. (2012). Action intentions modulate allocation of visual attention: Electrophysiological evidence. Frontiers in Psychology, 3. https://doi.org/10.3389/fpsyg.2012.00379

Wykowska, A., Schubö, A., \& Hommel, B. (2009). How you move is what you see: Action planning biases selection in visual search. Journal of Experimental Psychology: Human Perception and Performance, 35(6), 1755-1769. https://doi.org/10.1037/a0016798

Publisher's note Springer Nature remains neutral with regard to jurisdictional claims in published maps and institutional affiliations. 